Proc. Indian Acad. Sci. (Chem. Sci.), Vol. 96, No. 6, April 1986, pp. 473-498.

(C) Printed in India.

\title{
Why are certain substances metallic?
}

\author{
C N R RAO* and P P EDWARDS $\dagger$ \\ Solid State and Structural Chemistry Unit, Indian Institute of Science, Bangalore 560012 , \\ India \\ †Universiry Chemical Laboratory, University of Cambridge, Cambridge CB2 1EW, UK
}

\begin{abstract}
Metallic properties are by no means confined to elemental substances alone. $A$ variety of materials, both inor ganic and organic, show metallic properties. Some of these exotic substances exhibit electrical conductivities comparable to those of elemental metals like copper. A large number of systems traverse the transition from the metallic state to the nonmetallic state when there is a change in temperature, pressure or composition. Metal oxides provide a wide range of materials exhibiting metallic behaviour or going through the metal to non-metal $(M-N M)$ transition. Alkali metal-ammonia solutions, with which chemists are all too familiar, probably constitute one of the earliest and most widely studied examples of the $M-N M$ transition. However, a proper recognition of the metallization of ammonia in the context of the variety of solid systems exhibiting $M-N M$ transitions has only been possible recently. Another interesting class of substances is that of expanded metals such as $\mathrm{Hg}$ and $\mathrm{Cs}$ which become non-metallic when the density is reduced below a critical value. Several organic solids, metal-chain compounds and polymers are not only metallic, but also become superconducting at low temperatures. With such a galaxy of chemical substances exhibiting metallic behaviour, the fundamental, recurring question of vital interest is "what makes a metal?". In this contribution, we shall examine operational criteria as well as criteria derived from models to answer this question. A related question of equal interest to chemists is "how many atoms are necessary to bring about metallic properties?".
\end{abstract}

Keywords. Metal-non-metal transitions; metal-ammonia solutions; expanded metals; lowdimensional conductors; metallicity criteria.

\section{Introduction}

Metals have played a key role in man's life from time immemorial. A large majority of the elements on this planet are metallic under ambient conditions. Traditionally, lustre, high density and other characteristic physical properties have been associated with the metallic state and they are generaliy valid for solid elements such as copper and gold. Such notions met with unexpected difficulties when Davy discovered sodium and potassium in 1807, which possessed some of the properties attributed to metals but had extremely low densities. We also have metals such as mercury and cesium which are ordinarily liquids. More significantly, metallic behaviour is not confined to elements alone (Edwards and Rao 1985). Many metal oxides (e.g. $\mathrm{LaNiO}_{3}, \mathrm{ReO}_{3}, \mathrm{CrO}_{2}, \mathrm{TiO}$ ) and sulphides (e.g. $\mathrm{TaS}_{2}, \mathrm{NbS}_{3},(\mathrm{SN})_{x}, \mathrm{Sm}_{0.85} \mathrm{Gd}_{0.15} \mathrm{~S}$ ) exhibit metallic properties; some of them become superconducting at low temperatures. Metallic behaviour extends to organic solids as well and several organic 'metals' have been discovered in the last few years. There is also the fascinating class of solids which are non-metallic under

\footnotetext{
* To whom all correspondence should be addressed
} 
certain conditions but become metallic with change in temperature, pressure or composition (Mott 1974; Edwards and Sienko 1983; Edwards and Rao 1985). Typical examples of materials which exhibit such transitions with change in temperature or pressure are $\mathrm{Ti}_{2} \mathrm{O}_{3}, \mathrm{VO}_{2}, \mathrm{~V}_{2} \mathrm{O}_{3}, \mathrm{La}_{2} \mathrm{NiO}_{4}, \mathrm{LaCoO}_{3}, \mathrm{NiS}$ and SmS. Related series of compounds of the same transition metal exhibit a wide range of electrical and related properties as exemplified by the oxides of Ti, V and $\mathrm{Cr}$ (Rao and Subba Rao 1970; Goodenough 1971). $\mathrm{TiO}_{2}$ is an insulator while $\mathrm{TiO}$ is a metal; $\mathrm{Ti}_{2} \mathrm{O}_{3}$ and some members of the $\mathrm{Ti}_{n} \mathrm{O}_{2 n-1}$ family undergo metal to non-metal $(M-N M)$ transitions. $\mathrm{V}_{2} \mathrm{O}_{3}$ is an insulator while $\mathrm{VO}$ is a semi-metal: $\mathrm{Cr}_{2} \mathrm{O}_{3}$ is an insulator but $\mathrm{CrO}_{2}$ is a metal. Examples of solids traversing the $M-N M$ transition with change in composition include phosphorus-doped silicon and metal oxides such as $\mathrm{LaNi}_{1-x} \mathrm{Mn}_{x} \mathrm{O}_{3}$, $\mathrm{La}_{1-x} \mathrm{Sr}_{x} \mathrm{VO}_{3}$ and $\mathrm{Na}_{x} \mathrm{WO}_{3}$. Similar composition-controlled metal-non-metal transitions are exhibited by metal solutions in non-aqueous solvents (e.g. ammonia, methylamine). Then there are metals such as mercury and cesium which become insulating on expansion through a critical temperature and density.

Theoretical investigations to distinguish the metallic and the non-metallic states of solids have been made ever since the beginning of quantum mechanics and criteria for metallic character have been derived based on these studies. Attempts have also been made to relate atomic properties of elements in the free state with metallic or nonmetallic properties in the condensed state. In this article, we shall briefly present an overview of systems exhibiting metallic behaviour as well as those traversing the metal to non-metal transition in the condensed state and discuss the important criteria for metallic character. We shall also examine the various models for metal-non-metal transitions in solids and the question as to how many atoms make a metal.

\section{Operational criteria for metallicity}

Several operational criteria can be employed to distinguish metals from non-metals. For example, photo-electron spectra of metals clearly show a sharp rise in the density of states at the Fermi energy unlike non-metals. In figure 1 we show typical valence band spectra of some oxides to demonstrate how the spectra of metals are characteristically different from those of insulators. We cannot, however, employ the density of states and
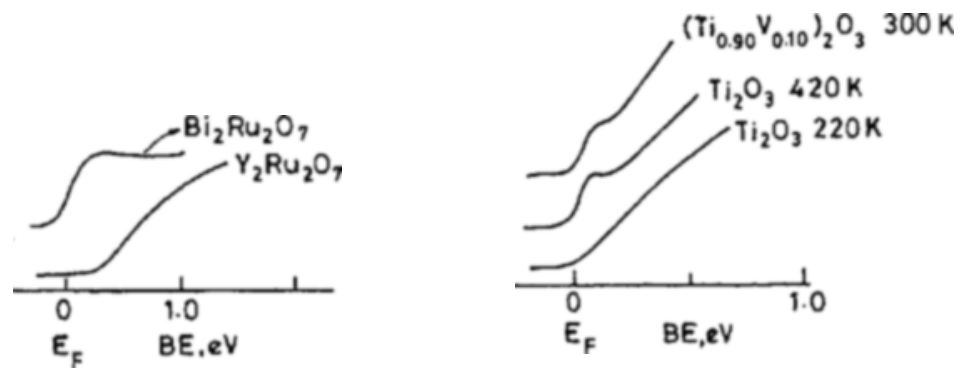

Figure 1. HeI photoelectron spectra of oxides in the valence band region showing the difference between metals and non-metals (Egdell and Goodenough 1983; Rao and Sarma 1982). $\mathrm{Bi}_{2} \mathrm{Ru}_{2} \mathrm{O}_{7}$ is a metal while $\mathrm{Y}_{2} \mathrm{Ru}_{2} \mathrm{O}_{7}$ is a non-metal. $\mathrm{Ti}_{2} \mathrm{O}_{3}$ becomes metallic above $410 \mathrm{~K} ;\left(\mathrm{Ti}_{0.90} \mathrm{~V}_{0.10}\right)_{2} \mathrm{O}_{3}$ is metallic. 
other properties related to the Fermi surface as criteria for metallic character since many metals are liquids. Magnetic properties can also be used to distinguish the metallic and the non-metallic states. For example, Pauli-paramagnetic behaviour is found in metals because of the presence of itinerant electrons while Curie behaviour is found in non-metals since they possess localized electrons. Similarly, optical properties; especially reflectance (lustre), can be used as a criterion. In fact, lustre has been the traditional characteristic attributed to metals such as gold. Reflectance spectra of metals show a sharp cut-off at the plasma frequency.

While the different considerations mentioned above provide a qualitative basis for describing metals, the best criterion appears to be electrical resistivity. The electrical resistivity of a metallic substance tends to a finite value (or zero in a superconductor) as the temperature tends to absolute zero, while in an insulator the resistivity tends to infinity. Accordingly, the electrical resistivity of a material very close to absolute zero provides a definitive operational criterion to distinguish metals from insulators. In figure 2 we show the electrical resistivity behaviour of a few oxides of transition metals to illustrate how 'metallic' some of them are compared to an elemental metal like copper. The resistivity behaviour of silicon samples doped with different concentrations of phosphorus is shown in figure 3 . The figure shows how the metal to non-metal

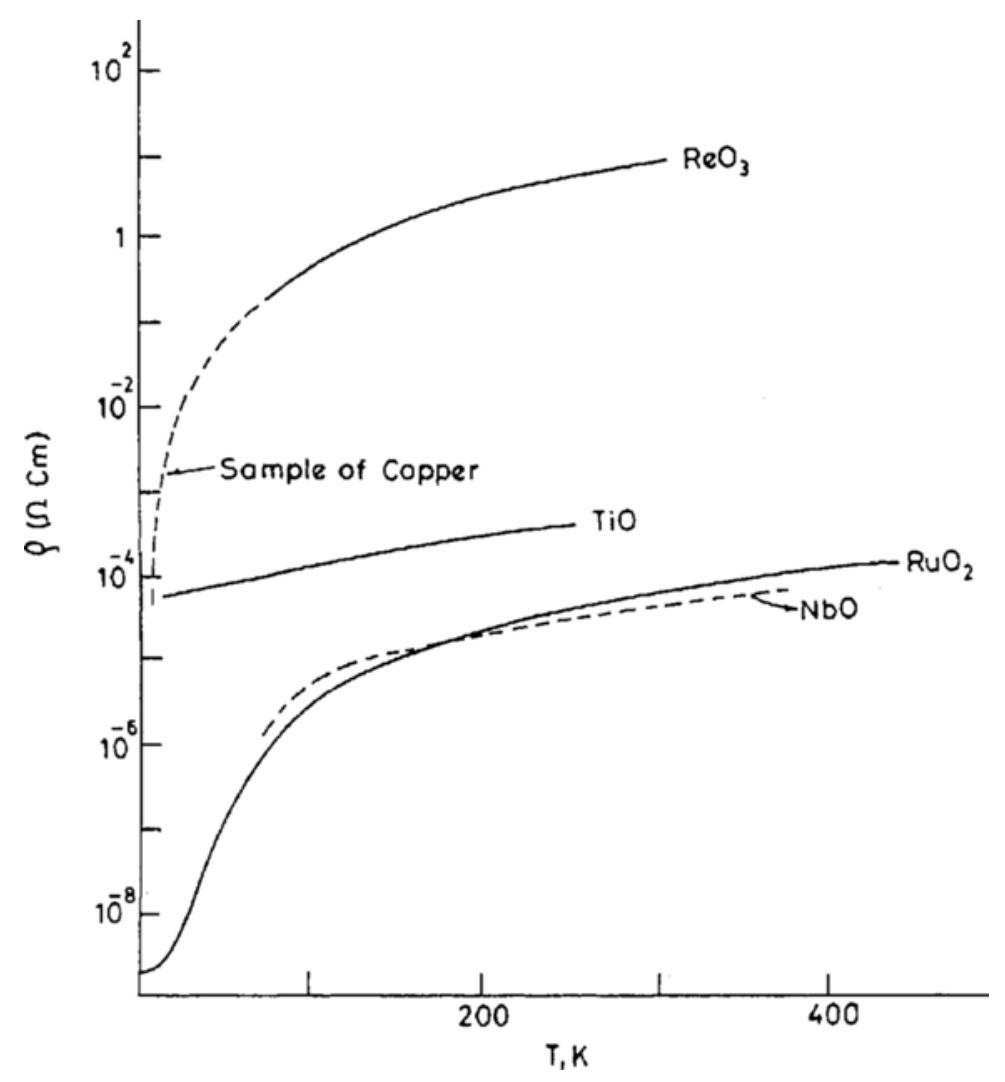

Figure 2. Electrical resistivity behaviour of a few oxide metals. 


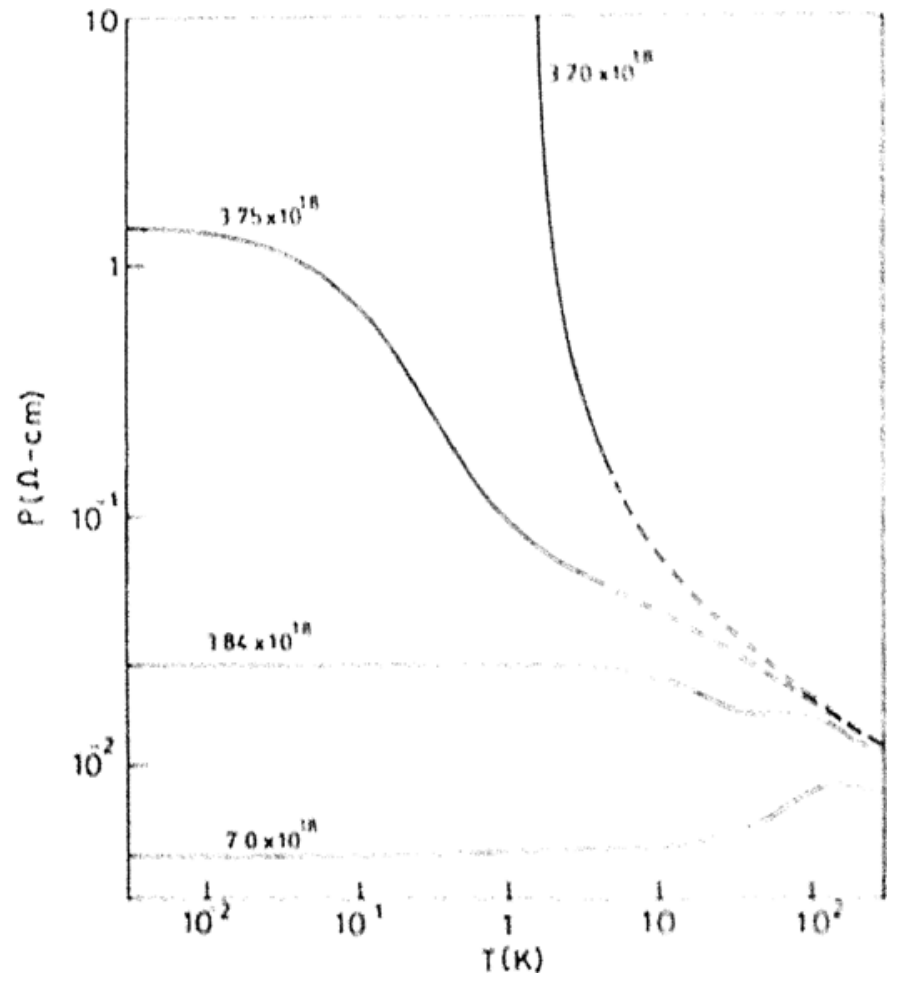

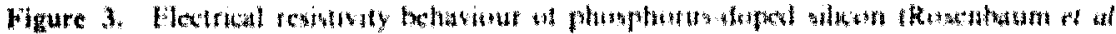

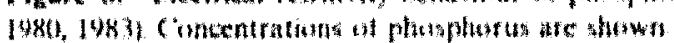

transition oceurs at a concentration of around $3.7 \times 10^{13}$ electrons cm ". In figure 4 we show how the temperature dependence of the electrical conductivity varies with the concentration of $\mathrm{Au}$ in the $\mathrm{Cs}_{1}, \mathrm{Au}_{\mathrm{x}}$ system (Ifensel 1480). Around $\mathrm{x}=0.44$ the temperature dependence of conductivity changes from negative to postive. In the insulating state, the alloy is best described as $\mathrm{C} \mathrm{s}^{* \mathrm{Au}}$. The change in sign of the temperature coefficient of resistivity undoubtedly provides a clear indication of the $M \sim N M$ transition in materials.

Based on these various operational criteria, chemists have distinguished metals from non-metals and have systematized elements in the periodic table 74 out of the 103

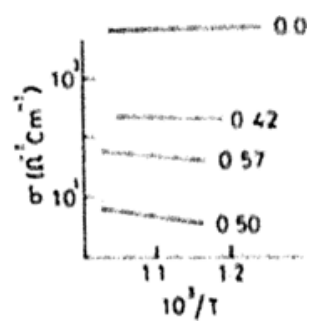

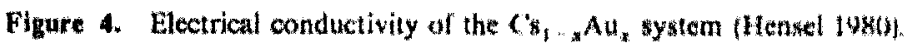




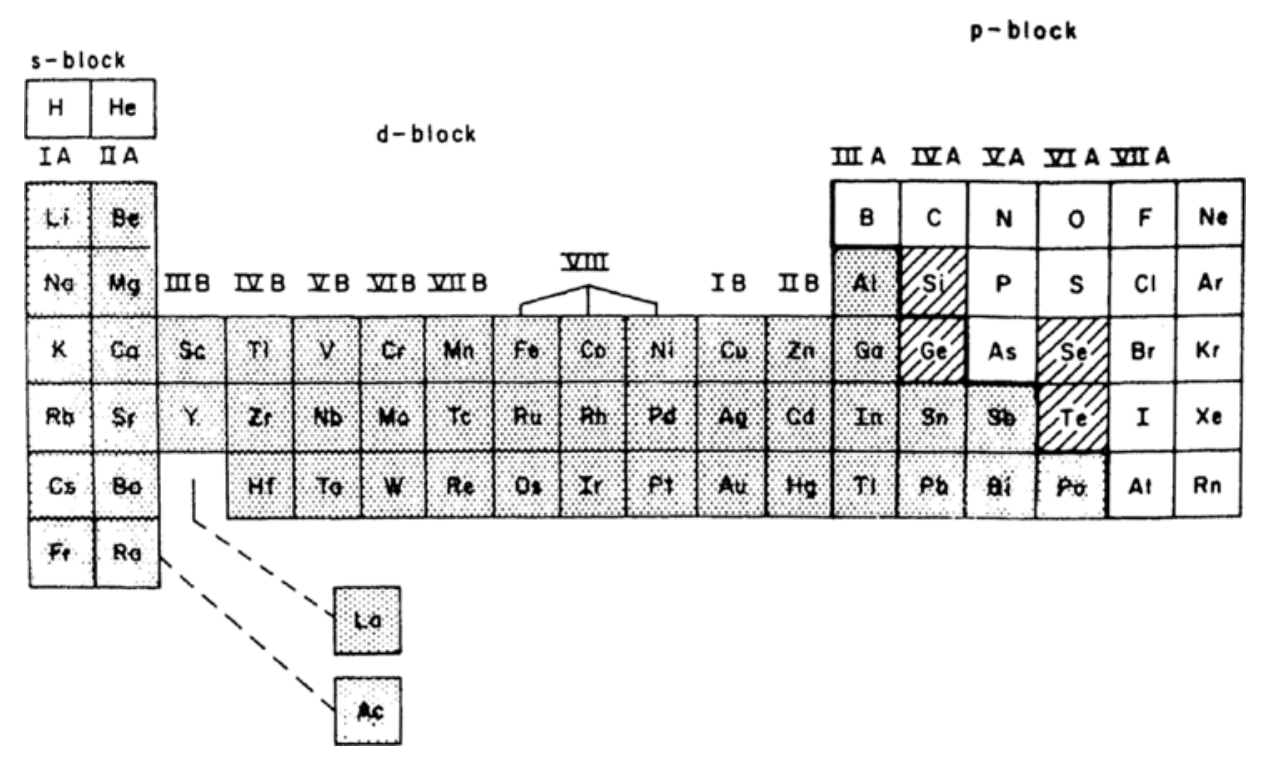

Figure 5. Periodic table showing metals and non-metals. Metals are shown in hatched squares. The clements $\mathrm{Si}, \mathrm{Ge}, \mathrm{Se}$ and $\mathrm{Te}$ become metallic in the molten state. $f$-block elements are not shown.

elements are metallic under ambient conditions, some of them being in the liquid state. In addition, $\mathrm{Si}, \mathrm{Ge}, \mathrm{Se}$ and $\mathrm{Te}$ become metallic in the molten state (figure 5). Many nonmetals including hydrogen become metallic on application of pressure. Thus, on Jupiter, all elements would be metallic under the ambient conditions of the planet.

\section{Systems exhibiting non-metal to metal transitions}

There are many materials (both elements and compounds) which exhibit transitions from the non-metallic to the metallic state and vice versa caused by changes in thermodynamic or compositional changes (Mott 1974; Edwards and Sienko 1983; Edwards and Rao 1985; Rao and Rao 1978). Such materials in the transition region between the metallic and non-metallic regimes are indeed most interesting. In table 1 we have listed representative examples of materials which go through $M-N M$ transitions to illustrate the variety of systems exhibiting this fascinating phenomenon.

Besides doped semiconductors (e.g. P doped Si) which show the $M-N M$ transition with change in dopant concentration, metal-rare gas films (e.g. Ar: $\mathrm{Na}$ and $\mathrm{Xe:} \mathrm{Pb}$ ) also show this behaviour. Some oxides transform from the non-metallic state to the metallic state with change in temperature; thus, $\mathrm{V}_{2} \mathrm{O}_{3}$ and $\mathrm{VO}_{2}$ show the transitions at $160 \mathrm{~K}$ and $340 \mathrm{~K}$ respectively. Application of pressure renders $\mathrm{V}_{2} \mathrm{O}_{3}$ metallic; dopants such as $\mathrm{Cr}$ and $\mathrm{Ti}$ also affect the $M-N M$ transition in $\mathrm{V}_{2} \mathrm{O}_{3}$ markedly. In systems such as $\mathrm{Na}_{x} \mathrm{WO}_{3}$ and $\mathrm{La}_{1-x} \mathrm{Sr}_{x} \mathrm{CoO}_{3}$, the material becomes metallic beyond a critical value of $x(x \gtrsim 0.3)$. An analogous liquid system is that of alkali metal-ammonia solutions where 
Table 1. Some examples of metallic and non-metallic states of matter.

\begin{tabular}{|c|c|c|}
\hline & Non-metallic & Metallic \\
\hline Elements & $\begin{array}{l}\mathrm{Si}(s) \\
\mathrm{H}_{2}(g)\end{array}$ & $\begin{array}{l}\mathrm{Si}(l) \text { at } T>2270 \mathrm{~K} \\
\mathrm{H}_{2}(s) \text { under high pressure }\end{array}$ \\
\hline Alloys & $C s_{1-x} A u_{x}(l)$ & $C s_{1-x} A u_{x}(l)$ \\
\hline Doped semiconductors & $\begin{array}{l}\mathrm{P}: \mathrm{Si}(s) \text { below critical concentration of } \\
\mathrm{P}\end{array}$ & $\begin{array}{l}\text { P: Si }(s) \text { above critical concentration of } \\
\text { P. }\end{array}$ \\
\hline Metal-rare gas films & $\begin{array}{l}\mathrm{Na}: \mathrm{Ar}(s) \\
\mathrm{Pb}: \mathrm{Xe}(s) \text { below critical concentration } \\
\text { of metal }\end{array}$ & $\begin{array}{l}\mathrm{Na}: \mathrm{Ar}(s) \\
\mathrm{Pb}: \mathrm{Xe}(s) \text { above critical concentration } \\
\text { of metal }\end{array}$ \\
\hline $\begin{array}{l}\text { Metal oxides } \\
\text { (all solids) }\end{array}$ & $\begin{array}{l}\mathrm{MgO} \\
\mathrm{V}_{2} \mathrm{O}_{3} \text { at } T<160 \mathrm{~K} \\
\mathrm{Na}_{x} \mathrm{WO}_{3}\left(x<x_{6}\right) \\
\mathrm{La}_{x} \mathrm{Sr}_{x} \mathrm{CoO}_{3}\left(x<x_{2}\right)\end{array}$ & $\begin{array}{l}\mathrm{ReO}_{3}, \mathrm{TjO} \\
\mathrm{V}_{2} \mathrm{O}_{3} \text { at } T>160 \mathrm{~K} \\
\mathrm{Na}_{x} \mathrm{WO}_{3},\left(x>x_{c}\right) \\
\mathrm{La}_{x} \mathrm{Sr}_{x} \mathrm{CoO}_{3}\left(x>x_{c}\right)\end{array}$ \\
\hline Organic solids & $\begin{array}{l}\text { Naphthalene, anthracene, }(\mathrm{CH})_{x} \text { pure } \\
\text { and or small dopant concentration } \\
\text { (dopant }=\mathrm{AsF}_{5}, \mathrm{ClO}_{4}, \mathrm{Na} \text { ) }\end{array}$ & $\begin{array}{l}\text { TIF-TCNQ, } \\
\left(\mathrm{CH}_{x}\right) \text { higher dopant concentration }\end{array}$ \\
\hline Solid polymers & Polyethelene, Pỵc & $(\mathrm{SN})_{x}$ organometallic polymers. \\
\hline Metals in solvents & $\mathrm{Na}$ in liquid $\mathrm{NH}_{3}\left(x<x_{c}\right)$ & $\mathrm{Na}$ in liquid $\mathrm{NH}_{3}\left(x>x_{c}\right)$ \\
\hline Expanded metals & $\begin{array}{l}\text { Liquids: } \mathrm{Hg}_{1} \mathrm{Cs} \text {, at high } T\left(T>T_{c}\right) \\
T_{c}=1750 \mathrm{~K}(\mathrm{Hg}) ; 1925 \mathrm{~K}(\mathrm{Cs})\end{array}$ & Liquids: $\mathrm{Hg}, \mathrm{Cs}\left(T<T_{\mathrm{r}}\right)$ \\
\hline $\begin{array}{l}\text { Expanded metal } \\
\text { compounds }\end{array}$ & $\mathrm{Li}\left(\mathrm{CH}_{3} \mathrm{NH}_{2}\right)_{4}$ & $\mathrm{Li}\left(\mathrm{NH}_{3}\right)_{4}, \mathrm{Ca}\left(\mathrm{NH}_{3}\right)_{6}$ \\
\hline
\end{tabular}

metallic behaviour is observed above a particular concentration of the metal. The case of expanded metals such as $\mathrm{Hg}$ and $\mathrm{Cs}$ was mentioned earlier. Having cited examples of $M-N M$ transitions, the question that we have to answer is 'When does a metal transform to a non-metal?'. Accompanying this query is the inseparable question 'What makes a metal?'. In what follows we shall attempt to answer these two fundamental questions in which all chemists should be interested.

\section{Theoretical models and criteria for metallization}

The earliest theoretical attempt to explain differences between the metallic and the nonmetallic states of solids was by Wilson who employed the free electron theory of Bloch (independent electron approximation). The Schrödinger equation for an electron moving in a periodic field of potential $U(x, y, z)$ is given by

$$
\nabla^{2} \psi+(2 m / \hbar)^{2}\{E-U(x, y, z)\} \psi=0
$$

where $\psi$ is of the form $\exp (i k r) U(x, y, z) ; U(x, y, z)$ has the period of the lattice and $k$ is the wave vector. This approach gives a description of the electron energy levels in terms of a family of continuous functions (bands), each possessing the lattice periodicity, giving rise to two distinct configurations: an insulator where the energy bands are full and a metal where the highest occupied band is only partially filled (figure 6). The model explains the electrical properties of simple metals including oxidic metals such as $\mathrm{ReO}_{3}$ (figure 7).

The simplistic Bloch-Wilson band picture of metals is, however, not universally valid. 


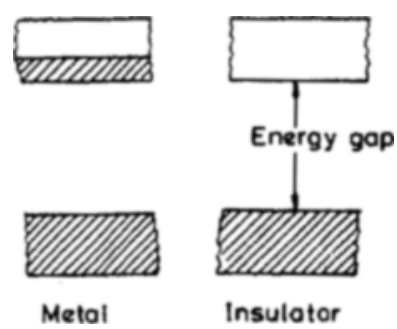

Figure 6. Band picture of a metal and an insulator.

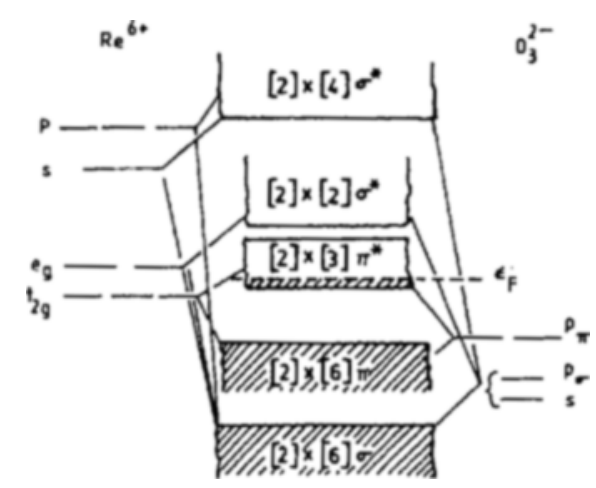

Figare 7. One-electron band diagram of $\mathrm{ReO}_{3}$ (Goodenough 1971).

For example, oxides such as $\mathrm{MnO}$ and $\mathrm{CoO}$ possessing the rocksalt structure are all insulators even though they have partially filled $d$-bands. At first sight this might not appear too surprising to chemists familiar with the localized-electron descriptions of transition metal complexes. However, TiO, on the other hand, is metallic and becomes a super-conductor below $2 \mathrm{~K}$. Curiously, the shortest metal-metal distance in TiO (along the diagonal in the (100) plane between the metal $t_{2 \beta}$ orbitals) is $2.94 \mathrm{~A}$ while in $\mathrm{MnO}$ and $\mathrm{CoO}$, it is 3.01 and $3.14 \mathrm{~A}$ respectively. Apparently, there is a critical metal-metal distance (or overlap) only below which these metal oxides become metallic (Goodenough 1971). The critical overlap integral or distance is larger than the metalmetal distance in insulating $\mathrm{MnO}, \mathrm{FeO}, \mathrm{CoO}$ and $\mathrm{NiO}$; in metallic TiO, the metal-metal distance is shorter than the critical distance while in VO, which is a semi-metal, they are nearly comparable.

The failure of the Bloch-Wilson scheme can be understood when we examine the case of an assembly of alkali atoms at different densities. At high densities, when the metalmetal separation is small, there is considerable overlap of the $6 \mathrm{~s}$ wave functions giving rise to the half-filled band responsible for the metallic behaviour. At small densities (large metal-metal distances), the Bloch-Wilson scheme would still predict metallic behaviour because of the presence of low-lying excited states and this is contrary to known facts. As shown in figure 8, the electrical conductivity of cesium traverses the $M-N M$ transition as the density (or the electron concentration) decreases. At low densities, electron-electron repulsion becomes significant just as in atoms; in the metal, this 


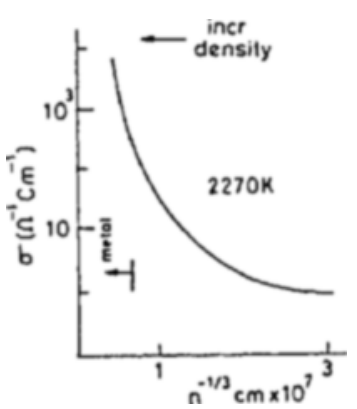

Figure 8. Electrical conductivity of cesium as a function of the cube root of the electron density (Hensel 1971). Metal-non-metal transition is indicated.

repulsion is reduced (screened) via the degenerate sea of electrons. Hubbard (1964) proposed a model which comes to grips with the problem of itinerant (band) and localized behaviour of electrons at high and low densities respectively.

According to the Hubbard model, the Coulomb repulsion energy, $U$, between two electrons on the same atom becomes more significant at low densities of the alkali metal (as in the atomic case) while the band width, $\Delta$, becomes the determining factor at high densities (figure 9). In the atomic limit, $U$ is the difference between the ionization energy and the electron affinity. The ratio $(\Delta / U)$ then provides a basis for distinguishing metals from non-metals (or insulators). According to this model, the condition for metallization is

$$
(\Delta / U)_{\text {critical }} \gtrsim 1 \cdot 2 \text {. }
$$

Based on dielectric screening properties of a uniform electron gas, Mott (1949) proposed a model to distinguish metals from non-metals. This model is independent of the atomic arrangement in the solid. The valence electrons are trapped in the coulomb field in the atomic state while in a metal the attractive interaction is screened (reduced) via the itinerant electrons. The screening potential is of the form,

$$
V(r)=\left(-e^{2} / r\right) \exp (-q r)
$$

where $r$ is electron-cation separation. The characteristic screening length $q^{-1}$ (which

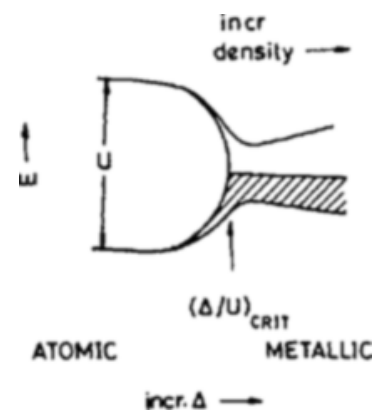

Figure 9. Metal-non-metal transition occurs at a critical value of $(\Delta / U)$ according to the Hubbard model (Hubbard 1964). 
depends on the electron density, $\boldsymbol{n}$ ) is rather small in metals. According to the model, there will be a first-order metal to non-metal transition (the Mott transition) when $V(r)$ has localized, bound states. The condition for such states to occur is given by

$$
n_{c}^{1 / 3} a_{H}^{*} \approx 0.25,
$$

where $n_{c}$ is the critical concentration for metallization and $a_{H}$ is the effective Bohr-orbit radius. Based on an analysis of extensive experimental data (see figure 10), Edwards and

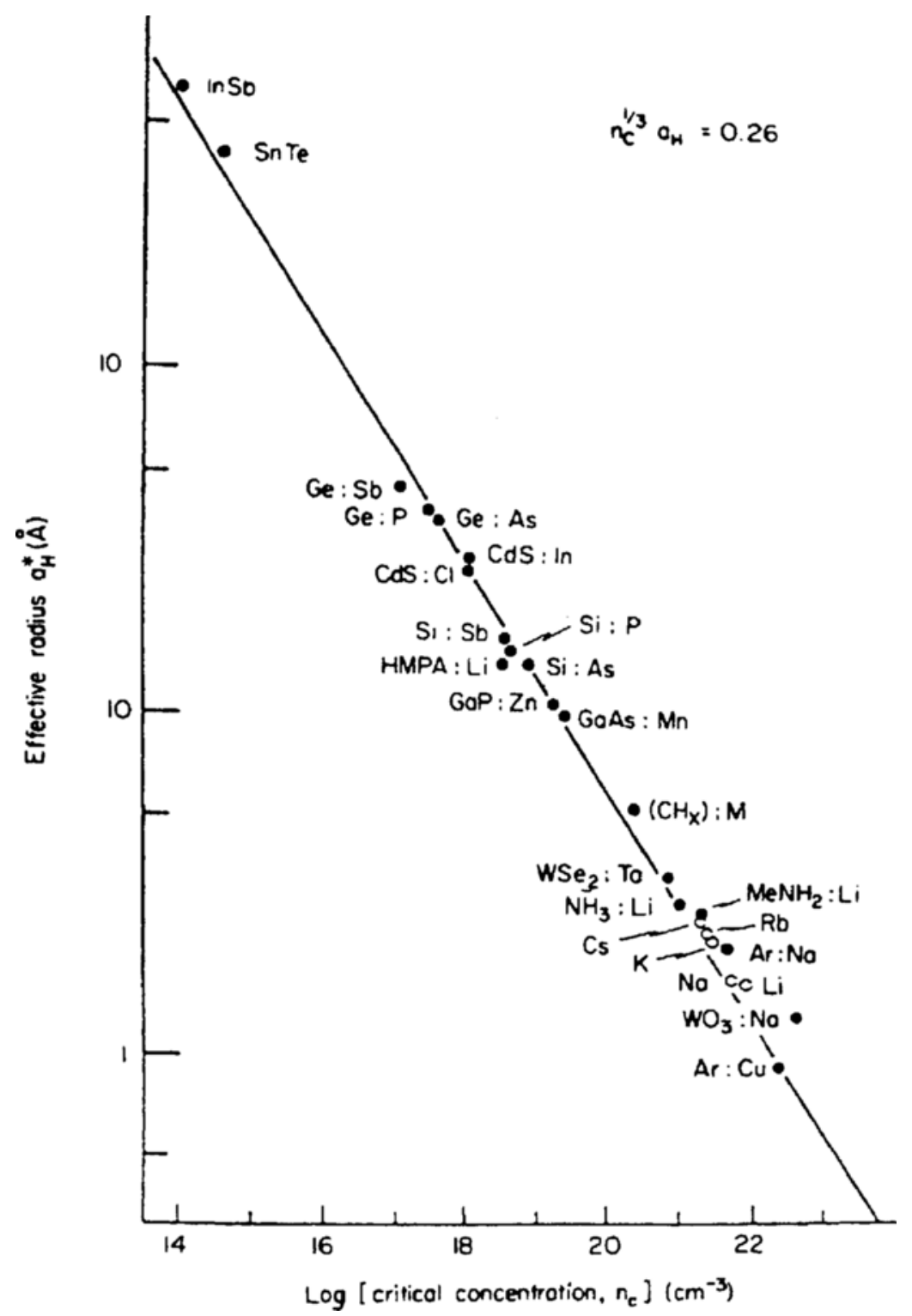

Figure 10. Logarithmic plot of the Bohr radius against the critical electron concentration for metallization (Edwards and Sienko 1981). 
Sienko (1981) have found that

$$
n_{c}^{1 / 3} a_{H}^{*} \approx 0.26 \text {. }
$$

One can consider the metal to non-metal transition to be essentially due to a competition between the kinetic and the potential energies of electrons. While the kinetic energy delocalizes electrons, potential energy localizes them (Ramakrishnan 1985). The condition for the kinetic energy to be greater than the potential energy is given by

or

$$
\left(h^{2} / m^{*}\right) n^{2 / 3} \gtrsim A\left(e^{2} / \varepsilon a_{H}^{*}\right)
$$

$$
n^{1 / 3} a_{H}^{*}=A \text {. }
$$

The best estimate of $A$ turns out to be 0.25 or $0-26$. The potential energy in the Hubbard model is the correlation energy $U$.

The Herzfeld criterion: Herzfeld (1927) proposed that local field corrections in an insulator drive it to become metallic when the insulator becomes sufficiently dense. The dielectric constant, $\varepsilon$, of such a system is expressed as

$$
\varepsilon=1+(4 \pi N \alpha) /[1-(4 \pi N \alpha / 3)] \text {, }
$$

where $\alpha$ is the polarizability of atoms. When $(4 \pi N \alpha / 3)=1, \varepsilon$ diverges. The above

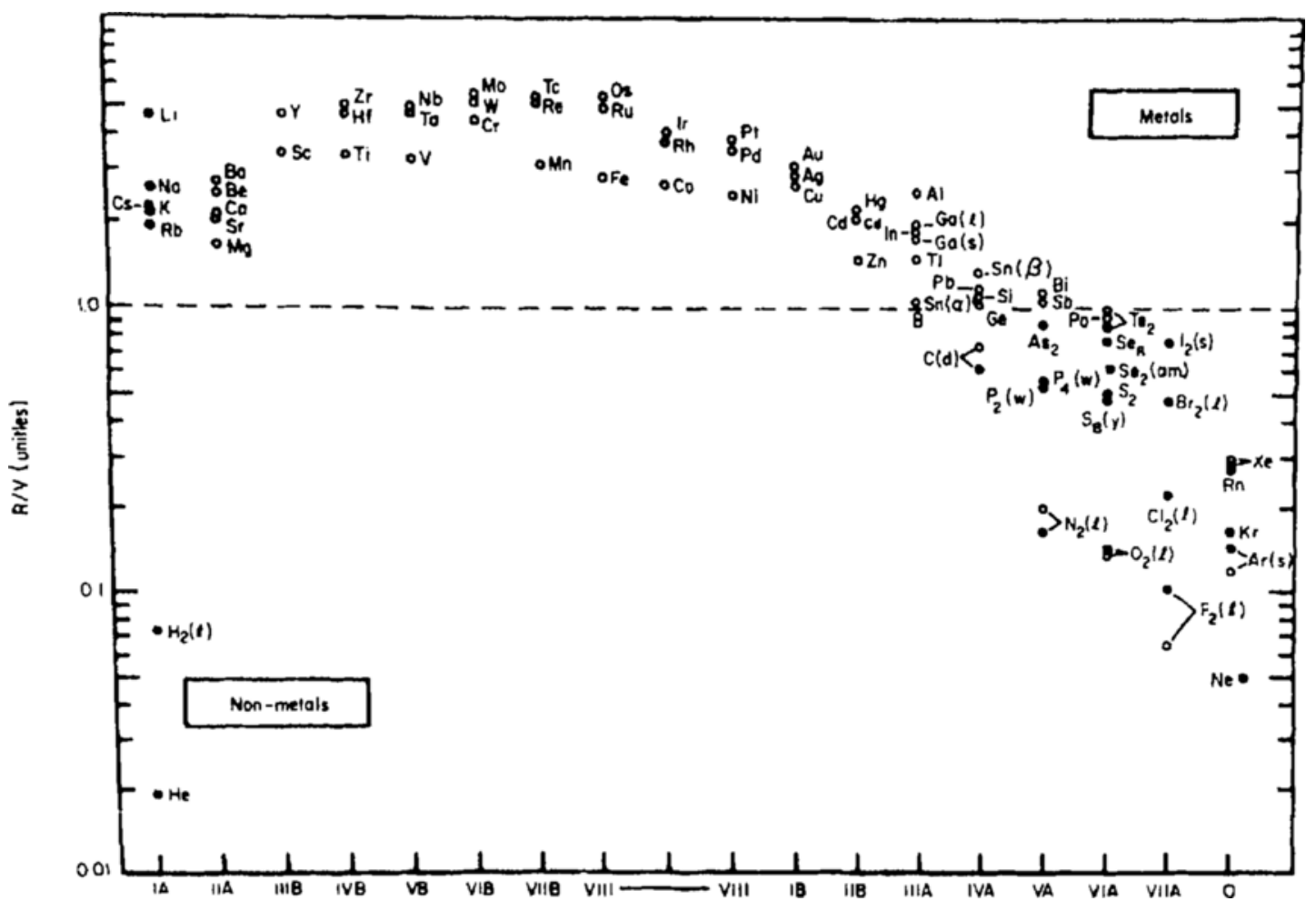

Figare 11. Metallization of elements in the periodic table as explained by the Herzfeld criterion (Edwards and Sienko 1982). 
equation in its simple form may not survive in dense systems (due to other processes contributing to $\alpha$ as atoms begin to overlap). According to Herzfeld, the characteristic frequency $v_{0}$ of the bound electrons in atoms is diminished at finite densities to

$$
v=v_{0}[1-(R / V)]^{1 / 2} \text {, }
$$

where $R$ is the molar refractivity of the atom (equal to $4 \pi N \alpha / 3$ ) in the gaseous state and $V$ is the molar volume in the condensed state. We readily see that the metal to non-metal transition should occur depending on whether $(R / V)$ is less than or greater than unity.

For a metal,

$$
(R / V) \geqslant 1,
$$

for an insulator,

$$
(R / V)<1 .
$$

There has been considerable interest in detecting the polarization catastrophe at the $M-N M$ transition. What is more pertinent to the discussion here is that the $(R / V)$ criterion is able to explain why most elements in the periodic table under ambient

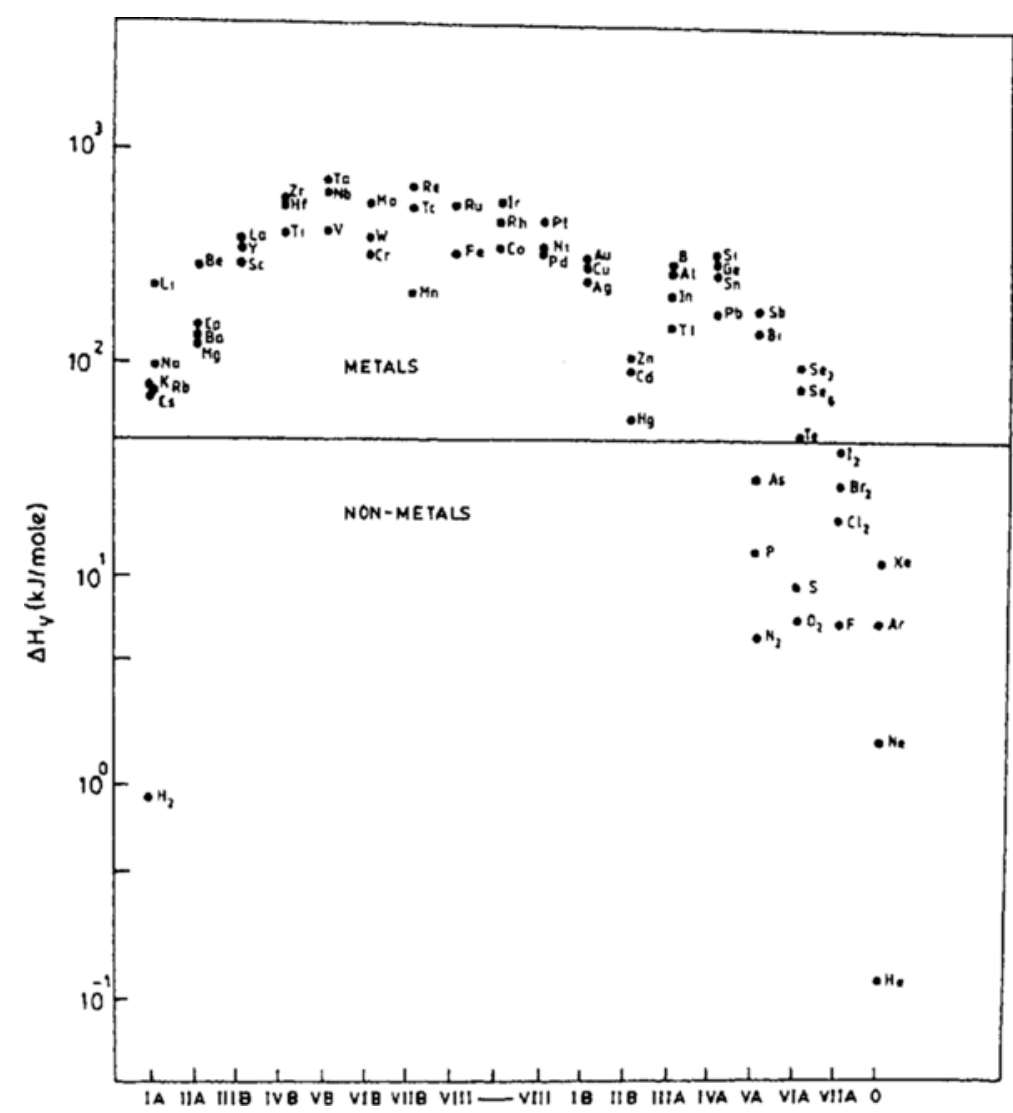

Figure 12. Latent heat of vapourization as a criterion for the metallic behaviour of elements (Rao and Ganguly 1986). 
conditions are metallic in the solid state (figure 11). The criterion predicts why hydrogen is metallic at high pressures; according to this criterion, mercury is metallic because of its high density in the liquid state.

A thermodynamic criterion: A thermodynamic criterion for metallization of elements has been proposed recently based on the latent heats of vapourization, $\Delta H_{v}$. Since more elements are metallic in the molten state and some metals are indeed liquid under ambient conditions, the liquid state of metals is probably more universal (at least 84 elements out of 103 being metallic in the liquid state). It is found that $\Delta H_{v}$ of metalic elements is greater than a critical value of $42 \mathrm{~kJ} \mathrm{~mol}^{-1}$ which demarcates metals from non-metals (Rao and Ganguly 1986) (figure 12). $\Delta \mathbf{H}_{v}$ is not only a simple operational criterion, but is perhaps superior in some respects to the Herzfeld criterion. Furthermore, it can be shown $\Delta H_{v}$ itself is proportional to $(R / V)^{n}$, thereby indicating the link between the two criteria. This also shows the important role of the atomic polarizability in determining the metallicity of elements.

\section{Different types of metal-non-metal transitions in solids}

Several kinds of metal-non-metal (M-NM) transitions occur in solids (Mott 1974; Rao and Rao 1978; Ramakrishnan 1985). We shall discuss them with illustrative examples.

(a) Mott transition: This transition, discussed in an earlier section, requires a firstorder transition (at $0 \mathrm{~K}$ ) between the localized and the itinerant electron regimes (Mott 1974,1949 ). Although the Mott criterion, $n_{c}^{1 / 3} a_{H}^{*} \approx 0.25$, describes the onset of metallic character in a large variety of solids over a wide range of critical electron concentrations $\left(\approx 10^{10}\right)$ and of the Bohr radii $(600 \mathrm{~A})$, the transition has not been found, in any simple solid, to be a function of temperature or pressure.

(b) Transitions occurring between extended states: These transitions can be described within the broad framework of the band theory of solids. The band structure of a solid made up of atoms with an even number of electrons can be made to change over to a situation where the empty and filled bands cross or overlap each other due to a change in pressure, temperature or suitable doping (Mott 1974; Rao and Rao 1978; Honig 1975). Such band-overlap or band-crossing transitions occur in solids such as $\mathrm{Ti}_{2} \mathrm{O}_{3}$, $\mathrm{VO}_{2}$ and $\mathrm{V}_{2} \mathrm{O}_{3}$ (figure 13) and they are generally accompanied by changes in crystal structure and, in some instances, in magnetic properties.

$\mathrm{Ti}_{2} \mathrm{O}_{3}$ (corundum structure) undergoes a second-order $M-N M$ transition with a 100-fold jump in electrical conductivity (figure 13) accompanied by a change in $c / a$ ratio around $410 \mathrm{~K}$. The structure remains the same throughout and the oxide is paramagnetic across the transition. Replacement of $\mathrm{Ti}$ by $\mathrm{V}$ up to $10 \%$ makes the oxide metallic and the $c / a$ ratio of the solid solution at the composition corresponds to that of the high temperature metallic phase. The $M-N M$ transition in $\mathrm{Ti}_{2} \mathrm{O}_{3}$, associated with only a small change in conductivity, arises from band crossing.

$\mathrm{VO}_{2}$ undergoes a first-order transition (monoclinic-rutile) accompanied by a ten thousand-fold jump in conductivity at $340 \mathrm{~K}$ (figure 13). The material remains paramagnetic throughout. This transition can be understood in terms of a crystal distortion model where the band gap opens up in the low temperature monoclinic phase.

$\mathrm{V}_{2} \mathrm{O}_{3}$ undergoes a first-order transition (monoclinic-corundum) accompanied by an unbelievable ten million-fold jump in electrical conductivity around $160 \mathrm{~K}$ (figure 13). 


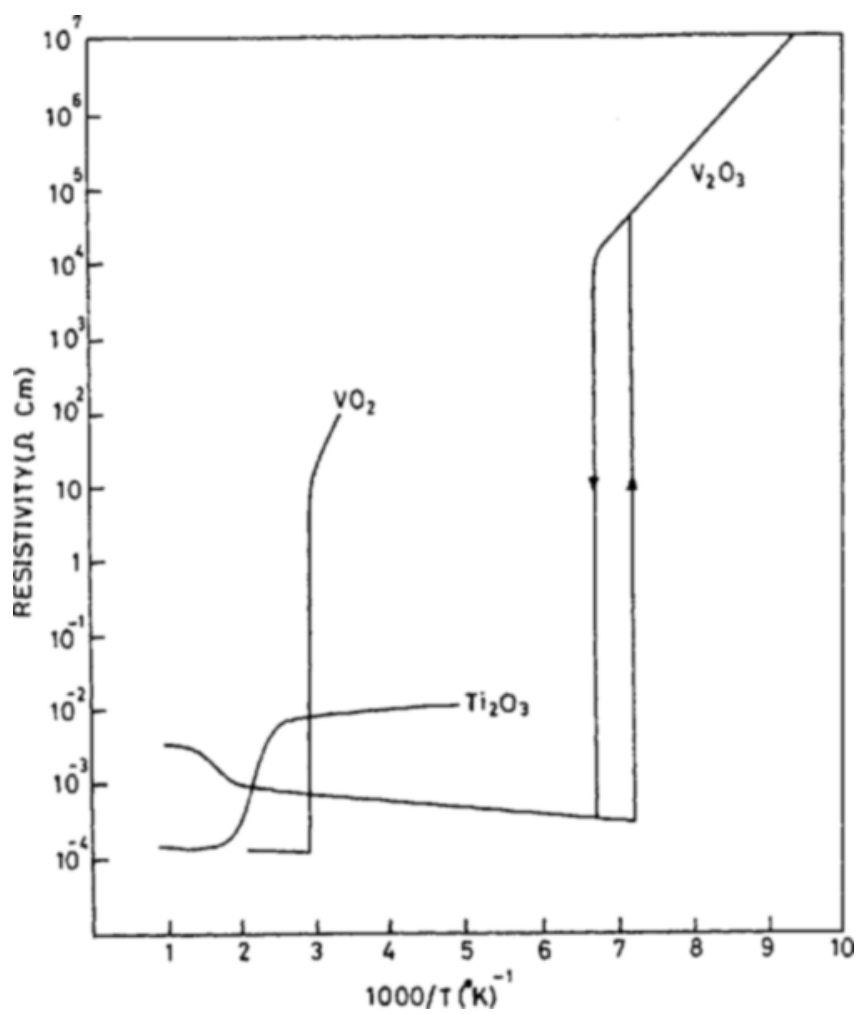

Figure 13. Metal-non-metal transition in $\mathrm{Ti}_{2} \mathrm{O}_{3}, \mathrm{VO}_{2}$ and $\mathrm{Y}_{2} \mathrm{O}_{3}$.

The transition is accompanied by a large change in $c / a$ ratio and volume, the $c / a$ ratio of $\mathrm{V}_{2} \mathrm{O}_{3}$ in the metalic $(M)$ phase being the highest amongst the oxides of corundum structure. At the transition, antiferromagnetic ordering changes to paramagnetic behaviour; the $160 \mathrm{~K}$ transition may thus be considered to be one from the antiferromagnetic insulating (AFI) phase to the $M$ phase. $\mathrm{V}_{2} \mathrm{O}_{3}$ also shows a second-order transition around $400 \mathrm{~K}$ with a small conductivity anomaly (figure 13). Application of pressure renders $\mathrm{V}_{2} \mathrm{O}_{3}$ metallic suggesting that this oxide is near the critical region. Accordingly, doping with $\mathrm{Cr}$ and Tí cause negative (increase in transition temperature) and positive (decrease in transition temperature) pressure effects; slight oxygen excess has the same effect as Ti doping (Honig 1982). Doping with small amounts of $\mathrm{Cr}(0.5$ to $2 \%$ ) changes the $400 \mathrm{~K}$ transition to a first-order transition from the metallic phase $(M)$ to an insulating, paramagnetic phase (I); the temperature of the $M-I$ transition decreases with increase in Cr concentration while that of the AFJ- $M$ transition increases. With further increase in temperature $(>400 \mathrm{~K}$ ), the $I$ phase transforms to a new highresistivity metallic phase, $M^{\prime}$. These varied features of the $M-N M$ transitions of $\mathrm{V}_{2} \mathrm{O}_{3}$ are truly fascinating and attempts have been made to understand them on the basis of theoretical models (Rao and Rao 1978; Honig 1982).

Besides the above three oxides, several other metal oxides (e.g. Magnéli phases $\mathrm{Ti}_{n} \mathrm{O}_{2 n-1} . \mathrm{NbO}_{2}$ ) and sulphides (e.g. $\mathrm{CrS}$ and NiS) also exhibit $M-N M$ transitions. The 
$M-N M$ transition in NiS is accompanied by an antiferromagnetic-paramagnetic transition and the latter is sufficient to explain the change in electrical conductivity.

$\mathrm{LaCoO}_{3}$ and other rare earth cobalt oxides exhibit a transition around $1200 \mathrm{~K}$ when the $e_{g}$ electrons of cobalt transform to a $\sigma^{*}$ band (figure 14). The transformation occurs over a wide range of temperature accompanied by an increase in the Co-O overlap; above the $1200 \mathrm{~K}$ transition, the temperature coefficient of conductivity indicates metallic behaviour (Bhide et al (1972). The value of resistivity at the transition temperature is barely $2000 \mu \Omega \mathrm{cm}$. It is interesting that the electrical resistivity changes of these cobalt oxides closely follow the changes in magnetic susceptibility.

It has been established recently (Rao and Ganguly 1985) that stoichiometric $\mathrm{La}_{2} \mathrm{NiO}_{4}$ possessing the $\mathrm{K}_{2} \mathrm{NiF}_{4}$ structure undergoes a $M-N M$ transition in two dimensions only ( $a b$ plane). The transition is due to the opening of a gap in the $\sigma^{*}$ band due to $x^{2}-y^{2}$ correlation effects.

(c) Valence fluctuation: SmS shows a discontinuous transition (decrease in volume) on application of pressure $(6.5 \mathrm{kbar})$ at room temperature due to the promotion of a $4 f$

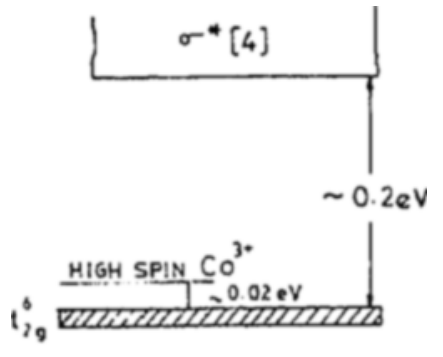

$T=O K$

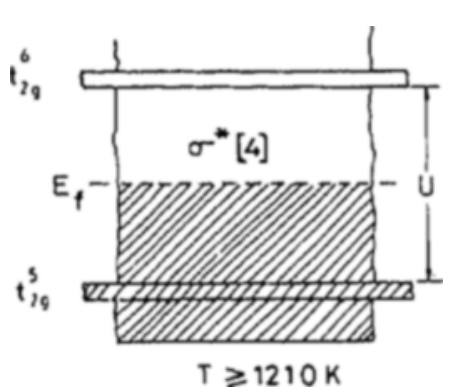

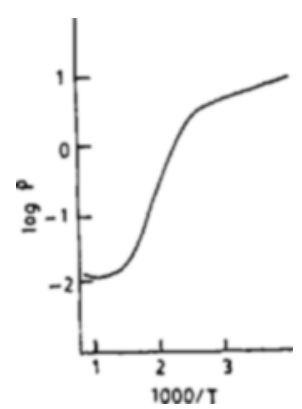

Figure 14. One-electron band picture of $\mathrm{LaCoO}_{3}$ at $0 \mathrm{~K}$ and above the $N M$-transition (Bhide et al 1972). Electrical resistivity behaviour is also shown.

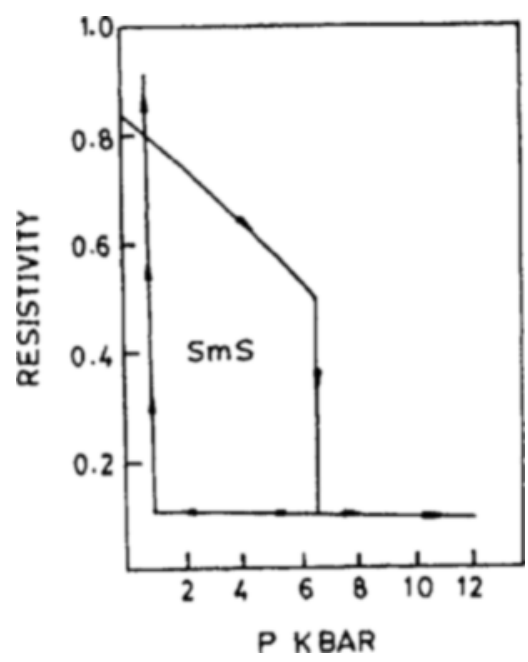

Figure 15. Electrical resistivity behaviour of $\mathrm{SmS}$ (Jayaraman et al 1970, 1973). 
electron to the conduction band. The change in volume is accompanied by a sharp decrease in resistivity (figure 15), but there is no change in structure type (Jayaraman et al 1970). In the high-pressure metallic phase, samarium is essentially 'trivalent'. Replacement of $\mathrm{Sm}$ by $\mathrm{Gd}$ as in $\mathrm{Sm}_{0.85} \mathrm{Gd}_{0.15} \mathrm{~S}$ stabilizes the high pressure metallic phase at room temperature and atmospheric pressure (Jayaraman et al 1970). On cooling this phase, there is an explosive first-order transition (due to increase in volume) to a non-metallic phase.

(d) Anderson transition: Anderson (1958) proposed that introduction of sufficient disorder in a solid can effectively transform the material from a metal to an insulator. Such a disorder-induced transition from an itinerant to a localized electron state (Ramakrishanan 1985) is referred to as the Anderson transition. According to Anderson, there is a critical value of $\left(\Delta / V_{0}\right)$ for which electron diffusion is impossible at $0 \mathrm{~K}$ and a nominally metallic system becomes insulating; here, $\Delta$ is the band width and $V_{0}$ is the random potential due to disorder. Mott (1972) has proposed that there is a finite minimum electrical conductivity. $\sigma_{\min }$, in a metallic three-dimensional disordered system. At $0 \mathrm{~K}$, one cannot have a non-zero value of conductivity less than $\sigma_{\min }$ :

$$
\sigma_{\min }=\left(\pi e^{2} / 4 z \hbar d\right)\left(\Delta / V_{0}\right)_{\mathrm{crit}}
$$
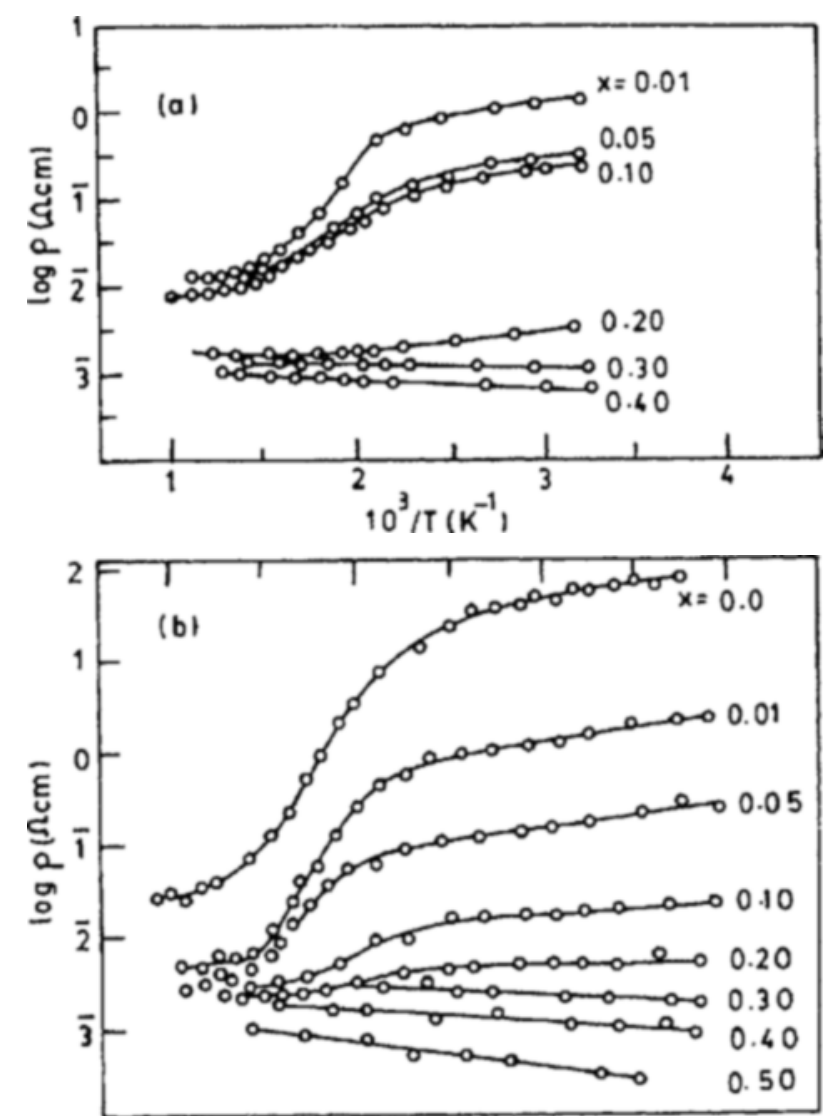

Figure 16. Electrical resistivity behaviour of $\mathrm{Ln}_{1}-\mathrm{Sr}_{x} \mathrm{CoO}_{3}[\mathrm{Ln}=\mathrm{Pr}$ or $\mathrm{Nd}]$ (Rao et al 1977). 
where $d$ is the distance between centres at the $M-N M$ transition. Although some of the early ideas of Mott on the mechanism of conduction in disordered solids have required some modification (Ramakrishnan 1985), the concept of minimum metallic conductivity has been found to be most useful. In fact, most experimental systems do exhibit $\sigma_{\min }$ behaviour at least above cryogenic temperatures. We shall examine the $\sigma_{\min }$ behaviour in some oxide systems where the $M-N M$ transition is brought about by compositional changes.

$\mathrm{LaCoO}_{3}$ and $\mathrm{LaVO}_{3}$ are both insulators at room temperature. However, replacement of $\mathrm{La}$ by $\mathrm{Sr}$ as in $\mathrm{La}_{1-x} \mathrm{Sr}_{x} \mathrm{BO}_{3}(B=\mathrm{Co}$ or $\mathrm{V})$ brings about remarkable changes in properties. In both these systems when $x \gtrsim 0.3$, the material becomes metallic (Rao and Ganguly 1985). At low $\mathrm{Sr}$ concentrations $(x<0 \cdot 1)$, the $B^{4+}$ holes are bound to $\mathrm{Sr}^{2+}$ while at high $x(>0-3)$ the holes form a degenerate gas; in the intermediate range of concentrations disorder arising from the random distribution of $\mathrm{Sr}^{2+}$ and $\mathrm{B}^{4+}$ ions induces localization of carriers. In figure 16 we show the electrical resistivity behaviour of $\mathrm{Ln}_{1-x} \mathrm{Sr}_{x} \mathrm{CoO}_{3}(\mathrm{Ln}=\operatorname{Pr}$ or $\mathrm{Nd})$. We clearly see that the $M-N M$ transition or the change in the sign of the temperature coefficient of resistivity (TCR) occurs around $3000 \mu \Omega \mathrm{cm}$ which corresponds closely to Mott's value of $\sigma_{\mathrm{min}}$.

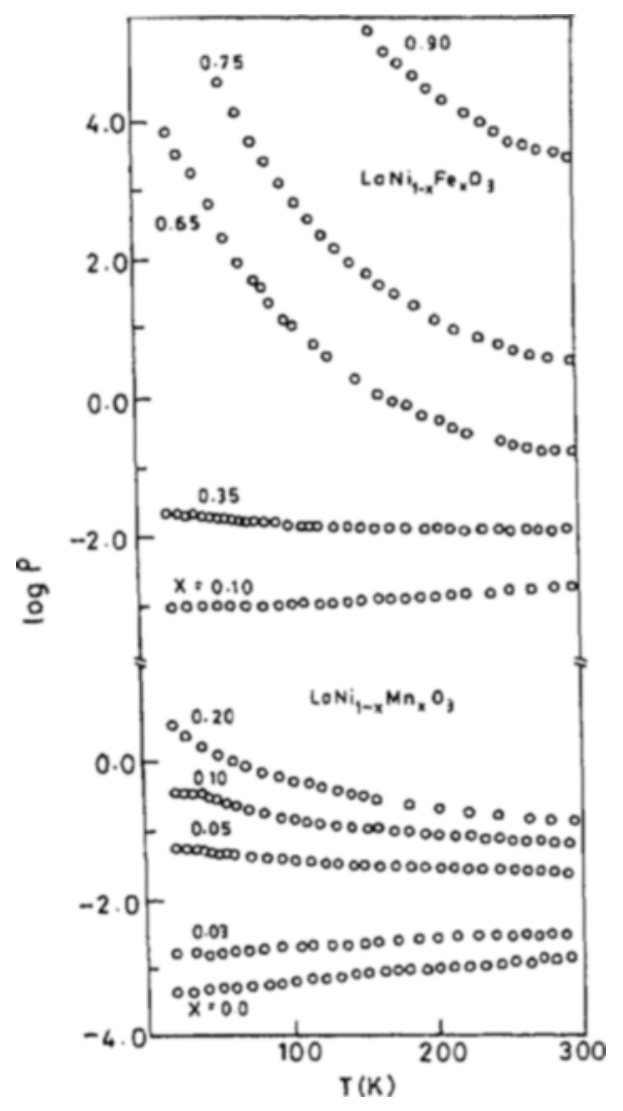

Figure 17. Electrical resistivity behaviour of $\mathrm{LaNi}_{2}{ }_{-x} \mathrm{M}_{x} \mathrm{O}_{3}[\mathrm{M}=\mathrm{Fe}$ or $\mathrm{Mn}]$ (Ganguly et al 1984). 
Recently, electronic and magnetic properties of the $\mathrm{LaNi}_{1-x} M_{x} \mathrm{O}_{3}(M=\mathrm{Cr}, \mathrm{Mn}, \mathrm{Fe}$ or Co) system have been investigated in detail (Vasanthacharya et al 1984; Ganguly et al 1984). $\mathrm{LaNiO}_{3}(x=0)$ is metallic and Pauli paramagnetic. Doping $\mathrm{LaNiO}_{3}$ with any of the $M$ ions makes it insulating beyond a critical value of $x$ as shown in figure 17 . Here again, the TCR changes sign around a resistivity value of $2000-3000 \mu \Omega \mathrm{cm}$. In all such oxide systems including bronzes such as $\mathrm{Na}_{x} \mathrm{WO}_{3}$, the TCR change occurs around a resistivity value $(2000-3000 \mu \Omega \mathrm{cm})$ which is about ten times the value of the resistivity where the TCR of amorphous metals changes sign (Mooij 1973; Rao and Ganguly 1985). This is not entirely surprising since the electron concentration in oxides is smaller than in amorphous metals. What is significant is that $\sigma_{\min }$ serves to demarcate the metallic and non-metallic regimes so successfully (Rao and Ganguly 1985).

\section{Metal clusters}

Alkali metal clusters are known to occur in the vapour phase and they have considerably lower ionization energies than isolated atoms. Today, we have several ways of preparing clusters of metal atoms of varying sizes. Metals such as cesium form larger clusters with increase in density (starting from isolated atoms in dilute vapours); as the density approaches a critical value, electrical conduction is facilitated (figure 8). The interesting question that one has to answer is "how many atoms are needed within a cluster to obtain metallic properties?'. Metallic conduction would require complete delocalization of electrons requiring a large number of atoms in the cluster, spin delocalization would, however, require a smaller number of atoms (Harrison and Edwards 1985). Metal particulates (as present on the surface of supported metal catalysts) constitute an aggregate somewhere between large metal atom clusters and the bulk metal. In figure 18 we have schematically depicted the atom, the cluster and the bulk metal regimes. While localized electron states would be present in discrete atoms and small clusters, quantum-size effects (Harrison and Edwards 1985) may well be found in large clusters.

In the last few years, a variety of compounds containing metal chusters bound to ligands (figure 19) have been synthesized (Lewis and Green 1982). In small-nuclearity cluster compounds the electrons are paired, but in high nuclearity cluster compounds electron levels are expected to be closely spaced. Magnetic susceptibility studies seem to indicate evolution of certain aspects of metallic behaviour in high nuclearity compounds (Johnson et al 1985). Metal cluster compounds also serve as model

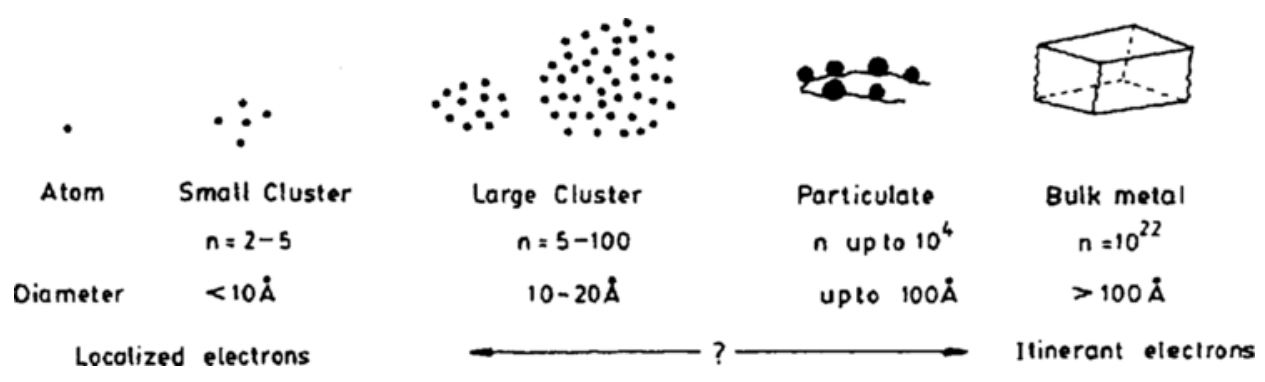

Figure 18. Metal atoms, metal clusters and bulk metal. 

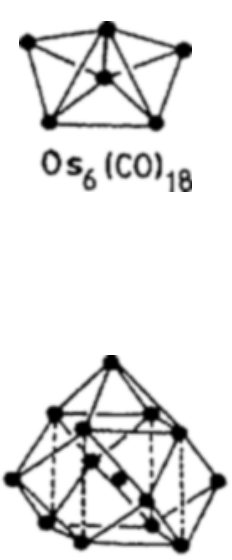

$\left[\mathrm{Rh}_{14}(\mathrm{CO})_{25}\right]^{4-}$

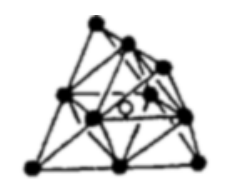

$\left[0 \mathrm{~s}_{10} \mathrm{C}(\mathrm{CO})_{24}\right]^{2-}$

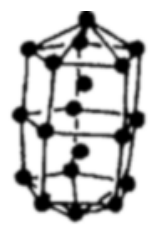

$\left[\mathrm{Pt}_{19}(\mathrm{CO})_{22}\right]^{4-}$

Figure 19. Typical metal cluster compounds.

compounds for surface species produced by the interaction of molecules with metal surfaces.

\section{Metal-ammonia solutions}

Solutions of alkali metals in liquid ammonia are blue and ionically conducting when dilute. In the bronze coloured, concentrated solutions, the electrical conductivity is in excess of that of liquid mercury. Over the intermediate composition range (1 to $7 \mathrm{~mol} \%$ metal*) a $M-N M$ transition takes place with quite striking changes in the electronic, thermodynamic and mechanical properties (Thompson 1976). As early as 1927, Herzfeld (1927) noted that the change in conductivity in sodium-ammonia solutions was a direct manifestation of the release of the metal valence electrons following a polarization catastrophe. It is now generally assumed (Edwards and Sienko 1981) that the Mott criterion (5) represents an accurate guide to the $M-N M$ transition in these systems. The metallization onset in sodium-ammonia solutions is also responsible for the remarkable liquid-liquid phase separation into metallic (bronze) and non-metallic (blue) constituents at temperatures below $\approx 243 \mathrm{~K}$.

As we have indicated earlier, a true experimental distinction between a metallic and a non-metallic system can only be rigorously applied at $T=0 \mathrm{~K}$. This is illustrated in figure 20 which shows 'zero temperature' (specifically $0.003 \mathrm{~K}$ ) conductivity studies on $\mathrm{Si}: \mathbf{P}$. The application of these ideas to the 'hot' sodium-ammonia solutions at $231 \mathrm{~K}$ is clearly tantalising but perhaps not as straightforward as at temperatures of a few

$*$ mol $\%$ metal $=\left(\frac{\text { moles of metal }}{\text { moles of metal + moles of } \mathrm{NH}_{3}}\right) \times 100$ 


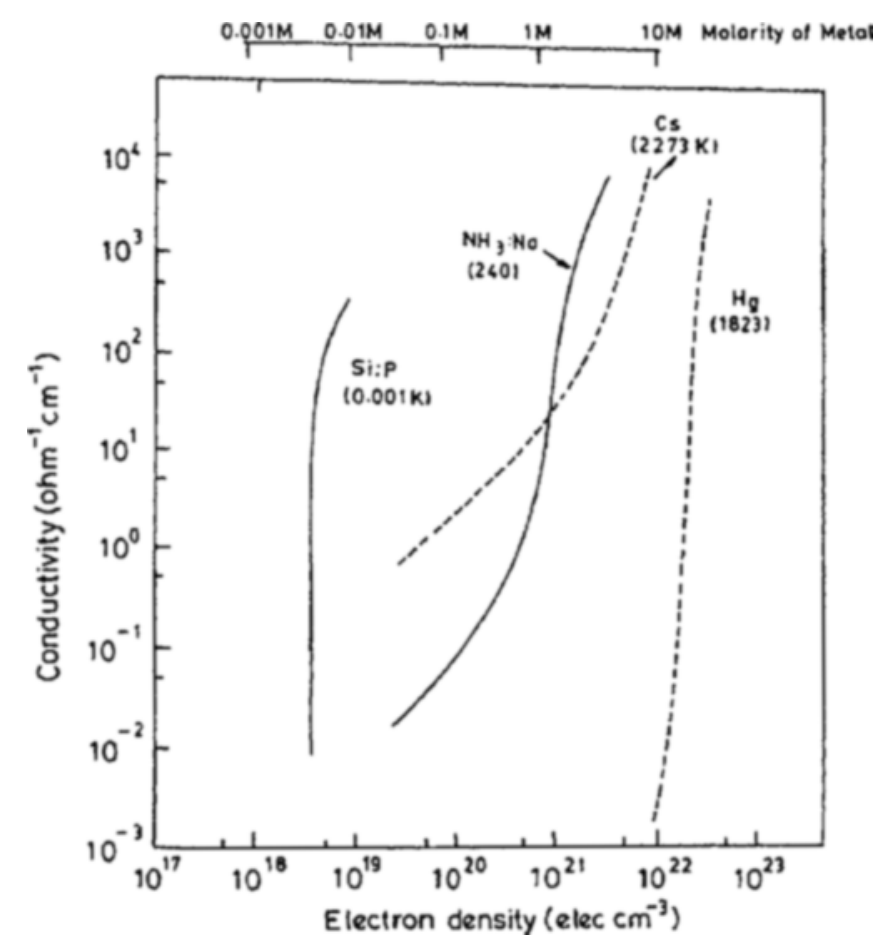

Figure 20. The metal-non-metal transition showing the concentration dependence of the electrical conductivity for a variety of systems. The appropriate observation temperatures are given.

millikelvin. However, note also from figure 20 that although extremely low temperatures generally produce a sharp $M-N M$ transition this is by no means a universal feature. The conductivity data for sodium-ammonia solutions $(231 \mathrm{~K})$ and expanded mercury (Hensel 1971) at $1823 \mathrm{~K}$ show a precipitous drop in conductivities for very small changes in electron density.

\section{Metal-metal halide melts}

Liquid metal-metal halide mixtures (e.g. $\mathrm{Na}-\mathrm{NaCl}$, Cs-CsCl, etc., $\mathrm{Hg}-\mathrm{HgCl}_{2}, \mathrm{Bi}-\mathrm{BiBr}_{3}$ ) form genuine solutions which link the metallic and non-metallic states by a continuous $M-N M$ transition at intermediate concentrations (Warren 1981). A number of physical mechanisms for electron localization versus itineracy have been involved which rely on the concepts of Mott, Hubbard and Anderson as applied to liquid state systems. Magnetic susceptibility and magnetic resonance studies (NMR, ESR) have proved to be most informative and direct indicators of how the microscopic electronic structure of both localized and itinerant electron states change across the transition. For example, the nuclear spin relaxation rate is governed by a characteristic time for fluctuations of the local magnetic field produced by the transient pairing of unpaired electrons (Warren 1981; Warren and Sotier 1981). Figure 21 shows the evolution of $\tau$ in liquid CsCsI solutions at $913 \mathrm{~K}$. From a value of $\tau \simeq 10^{-15} \mathrm{sec}$, characteristic of itinerant 


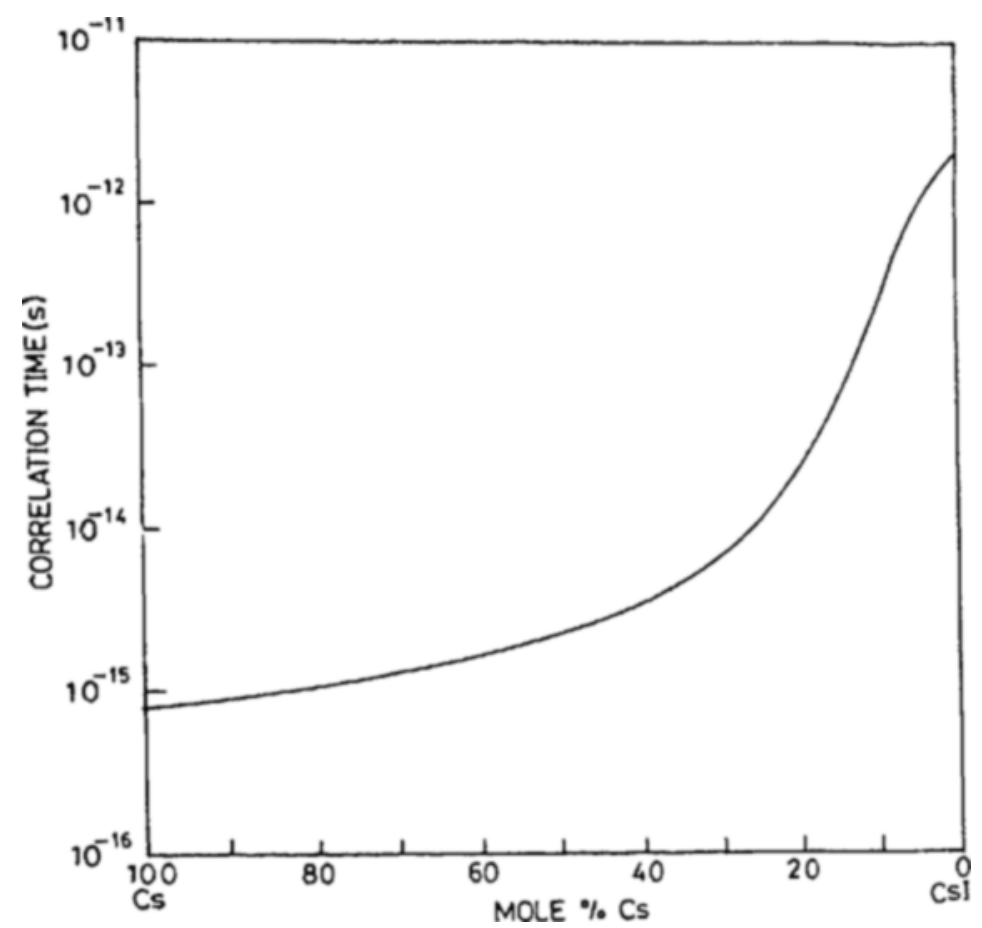

Figure 21. The characteristic NMR correlation time, $\tau$, versus composition for liquid Cs-CsI solutions (Warren and Sotier 1981) at $913 \mathrm{~K}$.

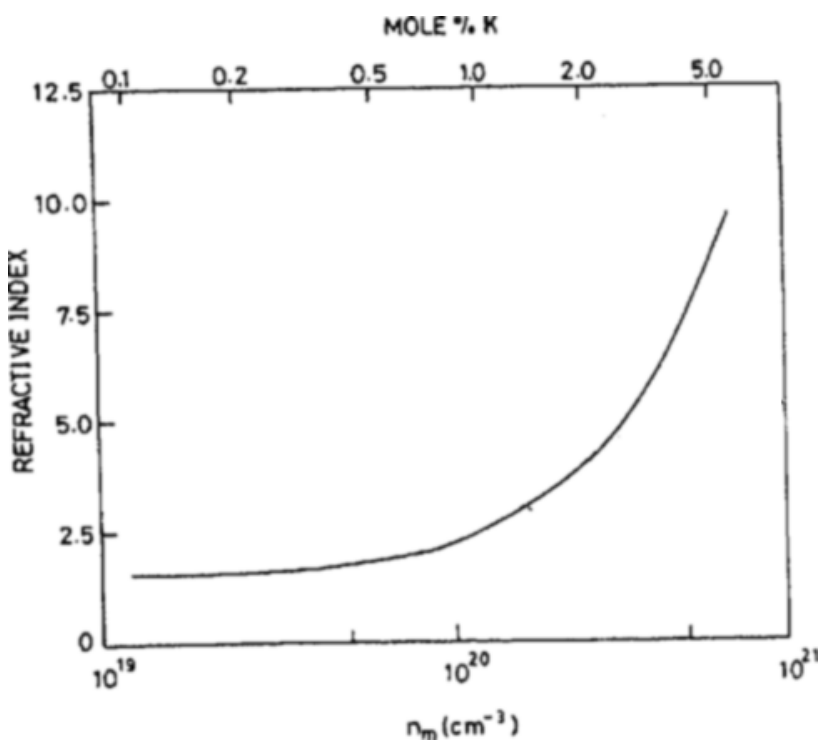

Figure 22. The refractive index vs. excess metal density $\left(n_{m}\right)$ for $\mathrm{K}-\mathrm{KCl}$ at $1133 \mathrm{~K}$. The upper scale indicates metal concentration in mol \% $\mathrm{K}$ (Freyland et al 1983). 
electrons in the pure metal, the correlation time increases monotonically to $\approx 10^{-12} \mathrm{sec}$ close to the non-metallic limit for which $\tau$ is now related to the normal ionic diffusion time (Warren and Sotier 1981).

Another approach for identifying the transition is the polarization catastrophe-the divergence of the refractive index on approaching the $M-N M$ transition from the insulating side. The onset of this effect has been observed by Freyland et al (1983) in $\mathrm{K} / \mathrm{KCl}$ (figure 22). Interestingly, the data show that the dielectric transition occurs at a substantially smaller value of the potassium concentration than either $\sigma_{\min }$ for the solutions or the critical point.

\section{Expanded metals}

Fluid metals, e.g. Cs, $\mathrm{Rb}, \mathrm{Hg}$, expanded to critical conditions offer perhaps the best possibility for an experimental verification of the various types of $M-N M$ transitions in one-component systems (Hensel 1971). Thus, the complexity of the concentrationinduced $M-N M$ transition in binary systems (e.g. metal-ammonia, etc.) can be avoided. However, a major technological challenge is posed not only by the very high temperatures and pressures required to bring the sample near its critical density (table 1), but also by the extremely reactive nature of the elements themselves. However, considerable, spectacular progress has been made during the past two decades into the study of the $M-N M$ transition in these expanded systems (Hensel 1984). Recent experiments on metals near their liquid-vapour critical points have shown that there are profound changes in their electronic structure in that region, as evidenced by dielectric anomalies and very rapid changes in electrical conductivity with density (Hensel 1984). Regardless of the precise details of the effective interactions in the limits of localized and itinerant electron regimes, the $M-N M$ transition ensures that these interactions must change with density. For example, there is a change from metallic cohesion in the dense liquid to a van der Waals type interaction between neutral atoms $\left(\mathrm{Cs}_{2}\right.$ etc.) in the

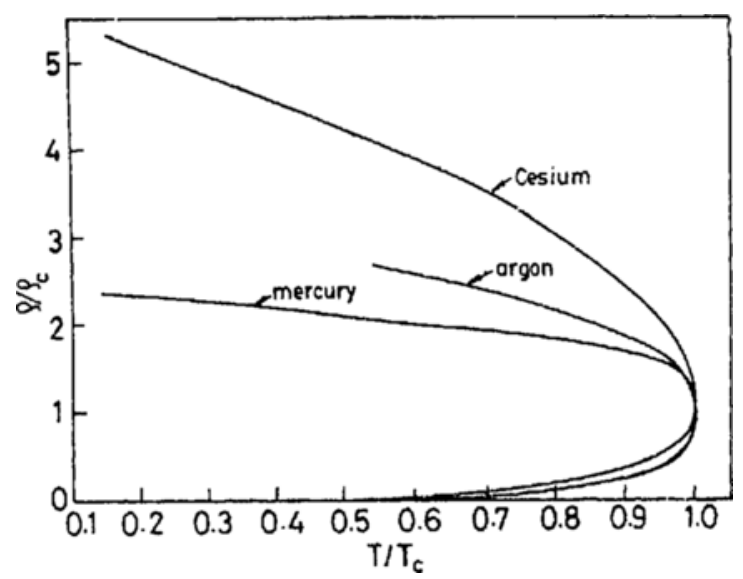

Figure 23. The density variation along the liquid-vapour coexistence curve for metals in comparison with argon (Hensel 1984). The reduced densities $P / P_{c}$ are plotted versus reduced temperature $T / T_{r}$. 
dilute vapour. This fundamental difference between the changing interactions taking place in an expanded liquid metal and what one might term a 'normal' fluid, such as argon, are manifest in the form of the liquid-vapour phase behaviour (figure 23). The phase diagram of the metals $\mathrm{Hg}$ and Cs have a distinct asymmetry in contrast to the phase behaviour of liquid argon. Thus 'Mott asymmetry' has also been noted for the coexistence curve for phase separation in sodium-ammonia solutions (Thomas 1984).

\section{Quasi-one-dimensional conductors}

A number of quasi-one-dimensional materials, both organic and inorganic, exhibit high electrical conductivity (Hodine 1984; Subramanyam 1981; Subramanyam and Naik 1984) and a present-day review of metallic materials would be incomplete without a reference to them. The conductivity is anisotropic since the electrons can move more easily in one of the directions. The popularity of this topic is primarily due to the expectation that high temperature superconductivity may be exhibited by them (Little 1964). Metallic and superconducting properties are not found in these materials as commonly because of the lattice distortion (Peierls' transition) that is expected to occur in one-dimensional metals or due to the presence of disorder. Quasi-one-dimensional conductors can be classified under the following categories: (a) organic charge-transfer complexes and salts, (b) conducting polymers and (c) metal-chain compounds. Some of these are depicted in figure 24.

A mong the organic metals, the most well known are charge-transfer complexes of the type, tetrathiofulvalene-tetracyanoquinodimethane (TTF-TCNQ) and salts such as TTF halides. A number of modifications of TTF-TCNQ including the selenium analogues have been investigated. Conditions for the formation of these metals are roughly the following: (i) the donor and acceptor molecules must be symmetrical; asymmetry leads to disorder effects; (ii) the molecules must be planar both in neutral and ionic states; a slight deviation in planarity could facilitate closer packing; (iii) the complex should form segregated stacks; this happens when the overlap between the donor and acceptor orbitals is not large; (iv) there must be unpaired electrons in the system and partial
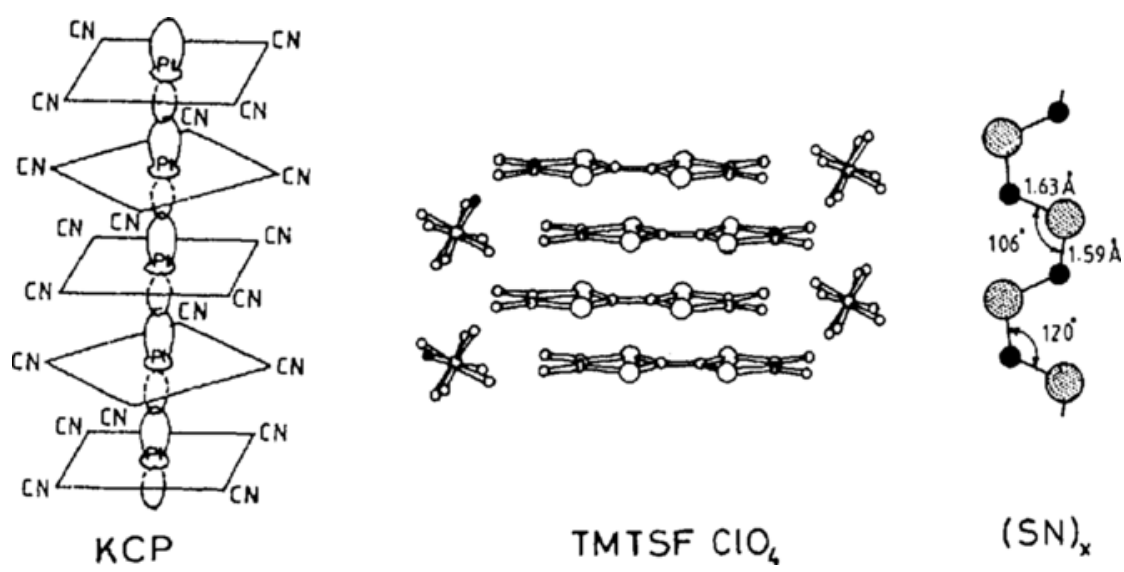

Figure 24. Typical quasi-one-dimensional conductors (see text for full description of the solids). 
charge-transfer must occur. Electrical conductivity is low in neutral and ionic complexes; ( $v$ ) the charge densities should be located near the opposite ends of the molecular unit so that it becomes highly polarizable.

Charge-transfer complexes are generally made up of stacks of donor and acceptor molecules. In single stack systems such as TTF halides and salts of TCNQ, only donor or acceptor molecules form stacks (figure 24). In TTF-TCNQ, the TCNQ stack is involved in conduction while in the corresponding selenium analogue both the donor and acceptor units are involved. Organic metals have resistivities in the range $5 \times 10^{-4}-10^{-2} \Omega \mathrm{cm}$. The metallic resistivity persists down to the lowest temperatures in some of the complexes (e.g. HMTSF-TCNQ where HMTSF = hexamethylene-tetraselenofulvalene) while in some others the resistivity goes through a minimum and then becomes semiconducting (e.g. TTF-TCNQ). Superconductivity has been observed in salts of tetramethyltetraselenofulvalene (TMTSF) under pressure; under normal pressure, the salts change from the metallic state to the non-metallic state. Only (TMTSF) ${ }_{2} \mathrm{ClO}_{4}$ becomes superconducting at atmospheric pressure. In figure 25 we show the phase diagram of ( $\mathrm{TMTSF})_{2} \mathrm{AsF}_{6}$ to illustrate the relation between the metallic, superconducting and non-metallic phases (Jerome and Schulz 1982).

Polysulphur nitride $(\mathrm{SN})_{x}$, is a conducting polymer (figure 20) which becomes superconducting at $0.3 \mathrm{~K}$ (Hatfield 1978). Halogenation of $(\mathrm{SN})_{x}$ increases the conductivity. Polyacetylene (Chien 1984) when suitably doped with electron acceptors or donors exhibits high conductivity (upto $10^{3} \Omega^{-1} \mathrm{~cm}^{-1}$ ); some of the dopants are $\mathrm{I}$, $\mathrm{AsF}_{5}$ and $\mathrm{Na}$. Electron conduction in polyacetylenes is explained on the basis of free radical states (neutral solitons), or positive and negative solitons (formed by addition of acceptors or donors respectively) as shown in figure 26. Recent studies (Soos and Ramasesha 1983) however indicate that correlation energy is the more important factor in giving rise to the energy gap in $(\mathrm{CH})_{x}$. Some other examples of conducting polymers are polyparaphylene- $\mathrm{AsF}_{5}$, polythienylene- $\mathrm{ClO}_{4}$ and poly-2,5-diylpyrrole- $\mathrm{BF}_{4}$ with conductivities in the range $10^{2}-10^{3} \Omega^{-1} \mathrm{~cm}^{-1}$.

Many metal chain compounds exhibit anisotropic electrical conductivity. $\mathrm{K}_{2} \mathrm{Pt}(\mathrm{CN})_{4} \mathrm{Br}_{0.3} 3 \mathrm{H}_{2} \mathrm{O}(\mathrm{KCP})$ which has a $\mathrm{Pt}$ - $\mathrm{Pt}$ distance close to that in platinum metal (figure 24) shows a $M-N M$ transition around $200 \mathrm{~K}$. Similar square planar complexes with Pt, Ir, Rh and Ni chains have been investigated (Krogman 1969; Miller 1982). Some of the metal trichalcogenides (e.g. $\mathrm{NbS}_{3}, \mathrm{TaSe}_{3}$ ) are one-dimensional

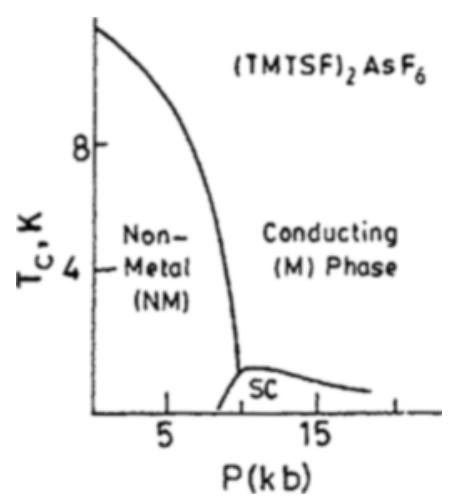

Figure 25. Phase diagram of superconducting (TMTsF) ${ }_{2} \mathrm{AsF}_{6}$ (Jerome and Schulz 1982). 

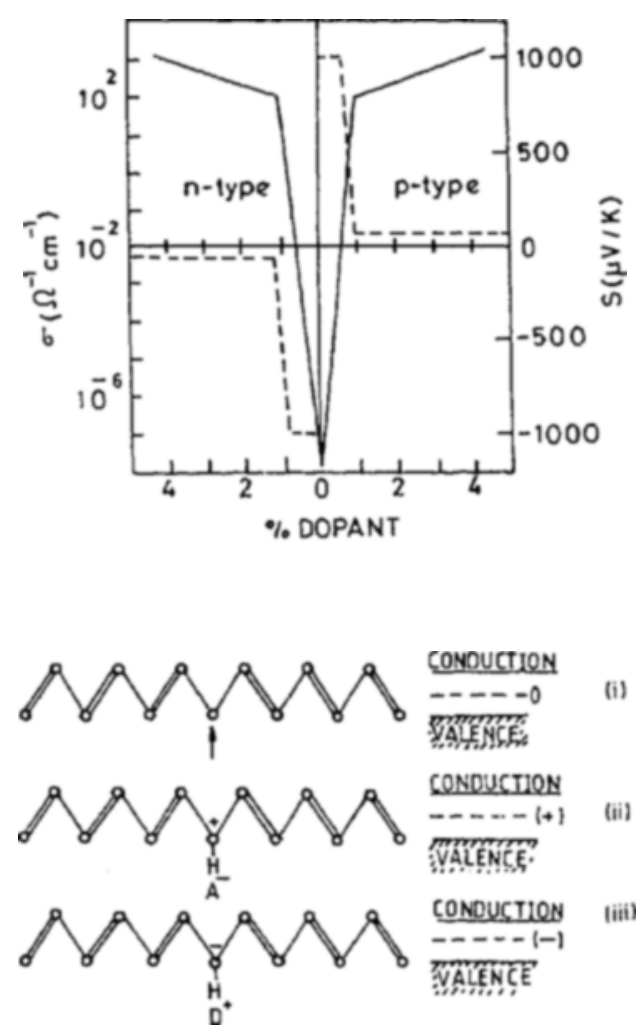

Figure 26. Electrical conductivity and thermopower (S) of doped polyacetylenes (Etemad $e t$ al 1982), (i) neutral soliton; (ii) positive soliton; (iii) negative soliton.

conductors which become superconducting at low-temperatures. In $\mathrm{Hg}_{2} \cdot{ }_{86} \mathrm{AsF}_{6}$, orthogonal strands of $\mathrm{Hg}$ are present and the $a b$ planes containing these strands exhibit metallic conductivity (Datars et al 1978). Other highly conducting systems of interest are organometallic polymers and metailoporphyrins.

\section{Concluding remarks}

In this article we have attempted to present a brief insight into the important and fundamental question: 'what makes certain materials metallic?'. With this in mind, we have outlined the vast variety of chemical systems which can exist either in metallic or non-metallic states. Our coverage has been primarily representative, but at the same time we have included aspects of both experiment and theory. It is our view that chemists play a cardinal role in the design and manufacture of materials. Our experierice has shown that chemists have generally been reluctant to come to grips with--indeed even to recognise-the seemingly strange symbolisms and concepts associated with the physics of itinerant electrons. They are, however, entirely happy with the content, formulation and application of models for localized electrons in liquids and solids. The converse would be applicable to the physics conmunity. 
We have identified and exemplified the transition region between the metallic and the non-metallic states of matter. The very fact that such a wide range of chemical substances can transform from a localized (non-metallic) to an itinerant (metallic) electron regime (table 1) seems to suggest that there must exist either a continuous or a discontinuous transition between the disciplines of chemistry and physics. Our hope is that the present article goes at least part of the way in understanding this fascinating transition.

\section{References}

Anderson P W 1958 Phys. Rev. 1091492

Bhide V G, Rajoria D S, Rao G R and Rao C N R 1972 Phys. Rev. B6 1021

Chien J C W 1984 Polyacetylene (New York: Academic Press)

Datars W R, Van Schyndel A, Lass J S, Chartier D and Gillespie R I 1978 Phys. Rev. Lett. 401184

Edwards P P and RaO CN R The metallic and the non-metallic states of matter (London: Taylor and Francis) 1985

Edwards P P and Sienko M J $1981 \mathrm{~J}$. Am. Chem. Soc. 1032967

Edwards P P and Sienko M J 1982 Acc. Chem. Res. 1587

Edwards P P and Sicnko M J 1983 Int. Rev. Phys. Chem. 383

Edgell R G, Goodenough J B, Hamnett A and Naish C C 1983 J. Chem. Soc. Faraday Trans. 79893

Etemad S, Heeger A J and MacDiarmid A G 1982 Ann. Rev. Phys. Chem. 33443

Freyland W, Garbade K and Pfeiffer E 1983 Phys. Rev. Lett. 511304

Ganguly P, Vasanthacharya N Y, Rao C N R and Edwards P P 1984 J. Solid State Chem. 54400

Goodenough J B 1971 Prog. Solid State Chem. 5149

Harrison M R and Edwards P P 1985 in The metallic and the non-metallic states of matter (eds) P P Edwards and C N R Rao (London: Taylor and Francis)

Hattield W E (ed.) 1978 Molecular metals (New York: Pienum Press)

Hensel F 1971 Ber. Bunsenges. Phys. Chem. 75619

Hensel F 1980 Angew. Chem., Int. Ed. Engl. 19593

Hensel F 1984 in NATO ASI series C. (eds) J V Acrivos, N F Mott and A D Yoffe (Dordrecht: Reidel) Vol. 30, p. 401

Herzfeld K F 1927 Phys. Rev. 29701

Hodine A J 1984 Syrith. Met. 9 B1, B97

Honig J M 1982 J. Solid State Chem. 451

Honig J M and Van Zandt L L 1975 Ann. Rev. Mater. Sci. 2225

Hubbard I 1964 Proc. R. Soc. London A281 401, and earlier papers

Jayaraman A, Bucher E, Dernier P D and Longinotti L D 1973 Phys. Rev. Lett. 31700

Jayaraman A, Narayanamurti V, Bucher E and Maines R G 1970 Phys. Rev. Lett. 25368,430

Jerome D and Schulz H J 1982 Adv. Phys. 31299

Johnson D C, Benfield R E, Edwards P P, Neison W 3 H and Vargas M D 1985 Nature (Londan) 314231

Krogman K 1969 Angew. Chem., Int. Ed. Engl. 835

Lewis J and Green M L H 1982 Philos. Trans. R. Soc. London A308 1

Little W A 1964 Phys. Rev. A134 1416

Miller J S (ed.) 1982 Extended linear chain compounds (New York: Plenum Press)

Mooij I H 1973 Phys. Status Solidi A17 521

Mott N F 1949 Proc. Phys. Soc. A62 416

Mott N F 1972 Plilos. Mag. 261015

Mott N F 1974 Metal-insulator rransitions (London: Taylor and Francis)

Ramakrishnan T V 1985 in The metallic and the nom-metallic states of matter (eds) P P Edwards and C N R Ran (London: Taylor and Francis)

Rao C N R, Buttrey D, Otsuka N, Ganguly P, Harrison H R and Honig J M 1984 J. Solid State Chem. 51266 Rao C N R and Ganguly $1985 \mathrm{a}$ in The netallic and non-metallic states of matter (eds.) P P Edwards and CN R Rao (London: Taylor and Francis)

Rao C N R and Ganguly P 1985b in Localization and metal-insulator transitions (eds) D Adler and H Fritsche (New York: Plenum Press) 
Rao C N R and Ganguly P 1986 Solid State Commun. 575

Rao C N R, Om Parkash, Bahadur D, Ganguly P and Nagabhushana 1977 J. Solid State Chem. 22353

Rao C N R and Rao K J 1978 Phase transitions in solids (New York: McGraw Hill)

Rao C N R and Sarma D D 1982 J. Solid State Chem. 4514

Rao C N R and Subbarao G V 1970 Phys. Status Solidi A1 597

Rosenbaum T F, Andres K, Thomas G A and Bhatt R N 1980 Phys. Rev. Lett. 451723

Rosenbaum T F, Milligan R F, Paalanen M A, Thomas G A, Bhatt R N and Lin W 1983 Phys. Rev. B27 7509

Soos Z G and Ramasesha S 1983 Phys, Rev, Lett. 512374

Subramanyam S V 1981 in Preparation and characterization of materials (eds) J M Honig and C N R Rao (New York: Academic Press)

Subramanyam $S$ V and Naik $H 1985$ in The metallic and the non-metallic states of matter (eds) P P Edwards and $C$ N R Rao (London: Taylor and Francis)

Thomas G A 1984 J. Phys. Chem. 883749

Thompson J C 1976 Electrons in liquid ammonia (Oxford: Clarendon Press)

Vasanthacharya N Y, Ganguly P and Rao C N R 1984 J. Solid. State Chem. 53140

Warren W W Jr 1981 in Advances in molten salt chemistry (eds) G Mamantov and J Braunstein (New York: Plenum Press) vol. 4 , p. 1

Warren W W Jr and Sotier $S 1981$ in Proceedings of the third international symposium on molten salts (eds) G Mamantov, M Blander and G P Smith (Princeton: Electrochemical Society) p. 95 